\title{
Blood urea nitrogen to creatinine ratio is associated with congestion and mortality in heart failure patients with renal dysfunction
}

\author{
Gaspare Parrinello $^{1}$ - Daniele Torres ${ }^{1} \cdot$ Jeffrey M. Testani $^{2} \cdot$ Piero Luigi Almasio $^{1}$. \\ Michele Bellanca ${ }^{1}$ - Giuseppina Pizzo $^{1} \cdot$ Francesco Cuttitta $^{1}$ - Antonio Pinto ${ }^{1}$. \\ Javed Butler $^{3} \cdot$ Salvatore Paterna ${ }^{1}$
}

Received: 5 March 2015 / Accepted: 13 May 2015/Published online: 3 June 2015

(C) SIMI 2015

\begin{abstract}
Renal dysfunction (RD) and venous congestion are related and common in heart failure (HF). Studies suggest that venous congestion may be the primary driver of RD in HF. In this study, we sought to investigate retrospectively the relationship between common measures of renal function with caval congestion and mortality among outpatients with $\mathrm{HF}$ and RD. We reviewed data from 103 HF outpatients (45 males, mean age 74 years, ejection fraction $41.8 \pm 11.6 \%$ ) with estimated glomerular filtration rate (eGFR) of $<60 \mathrm{ml} / \mathrm{min}$ in a single centre. During an ambulatory visit, all patients underwent blood test and ultrasonography of the inferior vena cava (IVC). Caval congestion was defined as IVC with both dilatation and impaired collapsibility. The best values of renal metrics in predicting caval congestion were determined with receiveroperating characteristic analysis. The $\mathrm{BUN} / \mathrm{Cr}$ ratio is moderately correlated with IVC expiratory maximum diameter $(r=0.31, p<0.0007)$. In a multiple logistic regression model, BUN/Cr $>25.5$ (adjusted OR 2.98, $p$ 0.015) and eGFR $\leq 45.8$ (adjusted OR 5.38, $p$ 0.002) identify patients at risk for caval congestion; a BUN/Cr $>23.7$ was the best predictor of impaired collapsibility (adjusted OR 4.41, p 0.001). a BUN/Cr $>25.5$ (HR 2.19,
\end{abstract}

Gaspare Parrinello

gaspare.parrinello@unipa.it

1 Dipartimento Biomedico di Medicina Interna e Specialistica, A.O.U.P. "Paolo Giaccone", Università degli Studi di Palermo, Piazza delle Cliniche 2, 90127 Palermo, Italy

2 Department of Internal Medicine and Program of Applied Translational Research, Yale University School of Medicine, New Haven, CT, USA

3 Cardiology Division, Stony Brook University, Stony Brook, NY, USA
$95 \%$ CI $1.21-3.94, p<0.001)$ and NYHA class 3 (HR $2.91,95 \%$ CI $1.60-5.31, p<0.0005)$ were independent risk factors associated with all-cause death during a median follow-up of 31 months. In outpatients with $\mathrm{HF}$ and RD, a higher $\mathrm{BUN} / \mathrm{Cr}$ and lower eGFR are reliable renal biomarkers for caval congestion. The $\mathrm{BUN} / \mathrm{Cr}$ is associated with long-term mortality and may help to stratify HF severity.

Keywords Heart failure - Renal dysfunction .

Outpatients · Congestion - Inferior vena cava $\cdot$ Collapse index

\section{Introduction}

Heart failure (HF) is a major public health problem, and its coexistence with renal dysfunction (RD) is common, and associated with a worse outcome. Both adverse structural and hemodynamic changes may explain this association [1]. Renal impairment in HF was traditionally assumed to be due to a decrease in cardiac output and a subsequent decrease in renal perfusion [2]. However, such studies suggest that venous congestion may be a primary driver of HF-induced RD more so than reduced cardiac output [3, 4].The natural history of HF may include recurrent decompensation where congestion plays a key role leading to HF progression and renal injury $[5,6]$.

Ultrasound of the inferior vena cava (IVC) and its collapsibility index have been demonstrated as a quantifiable and reliable approach for assessing volume status in HF [7, $8,23]$. This technique is aimed at identifying changes in the respiratory pattern of the IVC, thereby providing a dynamic metric for assessing volume status. Wide respiratory fluctuations of IVC collapsibility in $\mathrm{HF}$ may indicate an 
intravascular depletion, whereas low values indicate a higher probability of hemodynamic congestion, even in the absence of clinical signs. These inferences have been confirmed by various studies [7-10]. We hypothesized that $\mathrm{RD}$ is interrelated with intra-caval volume in chronic HF. In this study, we sought to determine the relationship between laboratory metrics of renal function and ultrasonographic indices of caval congestion in outpatients with clinically $\mathrm{HF}$ and RD.

\section{Methods}

\section{Patient population}

We retrospectively reviewed data of a cohort of patients referred to the outpatient $\mathrm{HF}$ clinic at University Hospital of Palermo. Study eligibility included consecutive patients with a diagnosis of HF according to European Society of Cardiology guidelines, [11] and concomitant RD as defined by estimated glomerular filtration rate $(\mathrm{eGFR})<60 \mathrm{ml} / \mathrm{min}$ according to the Modification of Diet in Renal Disease (MDRD) formula. To be included in the study, patients underwent clinical history, physical examination, blood chemistry analysis, and ultrasonography of the IVC at the same ambulatory visit. All subjects were also evaluated with cardiac ultrasonography. Patients hospitalized within 3 months for HF, acute coronary syndrome, cardiac tamponade, pericarditis, pulmonary thromboembolism, pleuropneumonia, acute renal insufficiency, pyelonephritis; patients requiring hospitalization for decompensated HF; patients with congenital heart disease, recipients of heart transplantation, chronic glomerulonephritis or on renal replacement therapy, chronic liver disease, blood and autoimmune diseases, cancer, and patients or those being treated with NSAIDs or steroids were excluded.

The study was conducted according to the Declaration of Helsinki and was approved by the Local Medical University Review Board. Patient records/information were redacted and de-identified prior to analysis.

\section{Renal function assessment}

Renal function was assessed by determining the serum creatinine, uric acid, blood urea nitrogen (BUN), and by calculating the eGFR and BUN to creatinine ratio (BUN/ $\mathrm{Cr}$ ). This ratio is a routinely available, inexpensive, noninvasive marker, which may serve as a tool to stratify patients with RD (normal ratio is 10:1-20:1) and for the differentiation of pre-renal RD from intrinsic renal parenchymal disease. This ratio increases due to a disproportionate tubular reabsorption of BUN compared to creatinine. In $\mathrm{HF}$, the BUN/Cr is elevated even when the kidneys are functioning properly. Creatinine clearance was determined by the four-variable MDRD formula: $186.3 \times$ creatinine $-1.154 \times$ age $-0.203 \times 1.212 \quad$ (if black) $\times 0.742$ (if female) [12].

\section{Inferior vena cava assessment}

The IVC was used as an index of central venous pressure as IVC measurements correlate with right atrial pressure [15]. IVC parameters were assessed by an experienced sonographer, blinded to the aim of the study, using an ultrasound machine (General Electric, Milan) and images were obtained in the semisupine position from an anterior trans-abdominal, sub-costal approach, using an abdominal convex probe $(3-5 \mathrm{MHz})$. The transverse diameter of the IVC was measured anterior to posterior at $2 \mathrm{~cm}$ from the IVC right atrial junction with the use of electronic callipers. Measurements were taken using M-mode at maximum diameter during expiration (IVCED) and minimum diameter during inspiration (IVCID). The collapse index (CI) was calculated as the percentage of the difference between maximum and minimum respiratory variation in diameter divided by the maximum: (IVCED - IVCID)/ IVCED $\times 100$. Patients with caval congestion were defined those with both increased IVCED $(>2 \mathrm{~cm})$ and impaired CI $(<50 \%)$ [16].

\section{Follow-up}

All patients were followed for a median follow-up of 31 months. The endpoint of the present follow-up investigation was defined as all-cause death. Mortality data were obtained from hospital medical records and/or telephone interviews with the patient or relatives.

\section{Statistical analysis}

Continuous variables are summarized as mean \pm standard deviation, and categorical variables as frequency and percentage. Intraobserver variations for IVC measurements were investigated using the Cohen's kappa test. Spearman correlation analysis and univariate linear regression analysis were used to assess the relationships between renal metrics and ultrasonographic indexes of IVC. Differences in the means were evaluated by an unpaired Student $t$ test, and the Chi squared test was applied to categorical variables. Receiver-operating characteristic (ROC) analysis was used to evaluate the best renal metric for predicting caval congestion. Significant univariate variables were included in the multivariate model. Multiple logistic regression was used to assess the adjusted odds of renal metrics for caval congestion, impaired collapsibility, and dilated expiratory diameter of IVC. Cox proportional hazard 
regression was used to analyse the factors associated with all-cause mortality, and hazard ratios (HR) and $95 \%$ confidence intervals (CI) were calculated. Kaplan-Meier analysis was used to illustrate outcome. All statistical analyses were performed with the Statistical Package of Social Sciences (SPSS Version 17, SPSS Inc, Chicago, IL, USA) and MedCalc package (Version 11.3, Belgium).

\section{Results}

\section{Patient population}

A total of 103 patients met the inclusion criteria and were included in the analysis. Baseline characteristics of the overall cohort are presented in Table 1. The mean age was $74.5 \pm 8.5$ and 58 patients $(56 \%)$ were female. NYHA functional class of the study population ranged from II to IV. Mean ejection fraction (EF) was $41.8 \pm 11.6 \%$ and 67 patients $(65 \%)$ had a reduced $\mathrm{EF}(<45 \%)$. In the entire

Table 1 Baseline characteristics of the study cohort expressed as mean \pm standard deviations

\begin{tabular}{ll}
\hline & Mean \pm SD \\
\hline Patients $(n)$ & 103 \\
Age (years) & $74.5 \pm 8.5$ \\
Sex M/F & $45 / 58$ \\
SBP (mmHg) & $130.4 \pm 19$ \\
DBP (mmHg) & $77.7 \pm 15.5$ \\
HR (bpm $\left.{ }^{\prime}\right)$ & $78.3 \pm 14.1$ \\
BUN (mg/dl) & $39.7 \pm 16.5$ \\
Creatinine $(\mathrm{mg} / \mathrm{dl})$ & $1.6 \pm 0.46$ \\
BUN/Cr & $24.6 \pm 8.1$ \\
eGFR (ml/min) & $36.8 \pm 10.4$ \\
Uric acid (mg/dl) & $7.5 \pm 2.8$ \\
Serum sodium (mEq/L) & $140.1 \pm 3.1$ \\
Haemoglobin (mg/dl) & $11.5 \pm 1.5$ \\
NYHA class 2 (pts) & 68 \\
NYHA class 3 (pts) & 28 \\
NYHA class $4(\mathrm{pts})$ & 7 \\
EF (\%) & $41.8 \pm 11.6$ \\
IVCED (mm) & $20.8 \pm 6.9$ \\
IVCID (mm) & $11.3 \pm 7.8$ \\
CI (\%) & $49.9 \pm 21.5$ \\
Furosemide (mg) & $238.6 \pm 177.7$ \\
Furosemide, $n(\%)$ & $95(92)$ \\
ACEI or ARB, $n(\%)$ & $90(88)$ \\
$\beta$-blocker, $n(\%)$ & $84(82)$ \\
Digoxin, $n(\%)$ & $26(25)$ \\
Spironolactone, $n(\%)$ & $31(30)$ \\
Allopurinol, $n(\%)$ & $51(50)$ \\
\hline & \\
&
\end{tabular}

cohort, mean values of serum creatinine, eGFR, BUN and $\mathrm{BUN} / \mathrm{Cr}$ were $1.6 \pm 0.46 \mathrm{mg} / \mathrm{dl}, 36.8 \pm 10.4 \mathrm{ml} / \mathrm{min} /$ $1.73 \mathrm{~m}^{2}, 39.7 \pm 16.5 \mathrm{mg} / \mathrm{dl}$ and $24.6 \pm 8.1$, respectively. In regard to the IVC, mean values of IVCED, IVCID and CI were $20.8 \pm 6.9 \mathrm{~mm}, \quad 11.3 \pm 7.8 \mathrm{~mm}$ and $49.9 \pm 21.5 \%$, respectively. Intraobserver agreement for assessment of IVC diameters was good $(k=0.621)$.

The above parameters of renal function and IVC indices were not different between patients with reduced $(\mathrm{rEF})$ and those with preserved EF ( $\mathrm{pEF})$.

\section{Renal function difference according to IVC parameters}

At an univariate model, patients with reduced IVC collapsibility ( $\mathrm{CI}<50 \%$ ), in comparison to patients with normal CI, had a significantly higher BUN/Cr $(27.3 \pm 9.2$ vs $22.2 \pm 6.2, p<0.001)$ and significantly lower eGFR $\left(37.8 \pm 10.9\right.$ vs $\left.42.3 \pm 11.7 \mathrm{ml} / \mathrm{min} / 1.73 \mathrm{~m}^{2}, p<0.05\right)$ (Table 2). Compared to patients with normal IVC diameter, those with dilated IVC had a significantly higher BUN (43.7 \pm 18.2 vs $37 \pm 14.9, p<0.05)$ and BUN/Cr $(26.7 \pm 9.2$ vs $23.1 \pm 7, p<0.025)$ and significantly lower eGFR $\left(37.8 \pm 10.9\right.$ vs $42.3 \pm 11.7 \mathrm{ml} / \mathrm{min} / 1.73 \mathrm{~m}^{2}$, $p<0.05$ ) (Table 2). Patients with caval congestion (presence of both reduced collapsibility and dilated IVC) had a significantly higher BUN (45.7 \pm 18.7 vs $36.8 \pm 14.7$, $p<0.01)$ and BUN/Cr $(28.2 \pm 9.3$ vs $22.9 \pm 7.0$, $p<0.002)$ and significantly lower eGFR (37.0 \pm 9.8 vs $42.2 \pm 12.0 \mathrm{ml} / \mathrm{min} / 1.73 \mathrm{~m}^{2}, p<0.02$ ) in comparison to patients with only one altered IVC parameter (Table 2). Regarding the serum creatinine and uric acid, no significant difference between the above groups was found, as well as age, left ventricular EF, mean blood pressure, furosemide dose and medications use.

\section{Relation between renal function and caval congestion}

Between metrics of renal function, the BUN/Cr was moderately and directly correlated with IVCED, suggestive of increased intravascular volume status $(r=0.33$, $p<0.0005)$, and poorly correlated with IVCID and CI, while BUN and eGFR were weakly correlated with IVCED (respectively, $r=0.23, p<0.009 ; r=-0.18, p<0.04$ ) and poorly correlated with IVCID and CI. Both serum creatinine and uric acid were not correlated with any parameters of IVC. Likewise, both $\mathrm{EF}$ and mean blood pressure were not correlated with any indices of IVC and renal function. Distinguishing patients according to $\mathrm{EF}$, BUN/Cr was the only renal parameter significantly correlated with IVCED in patients with preserved EF ( $r=0.52$, $p<0.0008)$. 
Table 2 Renal metrics, expressed as mean \pm standard deviations, in the study population sub-divided according to IVC parameters

\begin{tabular}{|c|c|c|c|c|c|c|c|c|c|}
\hline & $\begin{array}{l}C I \geq 50 \% \\
(n=56)\end{array}$ & $\begin{array}{l}\mathrm{CI}<50 \% \\
(n=47)\end{array}$ & $p$ & $\begin{array}{l}\mathrm{IVC} \leq 2 \mathrm{~cm} \\
(n=62)\end{array}$ & $\begin{array}{l}\text { IVC }>2 \mathrm{~cm} \\
(n=41)\end{array}$ & $p$ & $\begin{array}{l}\text { No CC } \\
(n=66)\end{array}$ & $\begin{array}{l}\mathrm{CC} \\
(n=37)\end{array}$ & $p$ \\
\hline Age ( & $73.8 \pm 7.7$ & $75.2 \pm 8.2$ & NS & $73.5 \pm 7.8$ & $75.8 \pm 8$ & NS & $74.1 \pm 8.0$ & $75.2 \pm 7.9$ & NS \\
\hline Sex M/F & $24 / 32$ & $21 / 26$ & NS & $27 / 35$ & $18 / 23$ & NS & $28 / 42$ & $17 / 16$ & NS \\
\hline Creatinine (mg/dl) & $1.6 \pm 0.5$ & $1.5 \pm 0.3$ & NS & $1.6 \pm 0.5$ & $1.6 \pm 0.4$ & NS & $1.6 \pm 0.5$ & $1.6 \pm 0.4$ & NS \\
\hline BUN (mg/dl) & $36.9 \pm 14.9$ & $42.8 \pm 17.9$ & NS & $37 \pm 14.9$ & $43.7 \pm 18.2$ & $<0.05$ & $36.8 \pm 14.7$ & $45.7 \pm 18.7$ & $<0.01$ \\
\hline $\mathrm{BUN} / \mathrm{Cr}$ & $22.2 \pm 6.2$ & $27.3 \pm 9.2$ & $<0.001$ & $23.1 \pm 7$ & $26.7 \pm 9.2$ & $<0.025$ & $22.9 \pm 7.0$ & $28.2 \pm 9.3$ & $<0.002$ \\
\hline eGFR (ml/min) & $42.3 \pm 11.7$ & $37.8 \pm 10.9$ & $<0.05$ & $42.3 \pm 11.7$ & $37.8 \pm 10.9$ & $<0.05$ & $42.2 \pm 12.0$ & $37.0 \pm 9.8$ & $<0.02$ \\
\hline Uric acid $(\mathrm{mg} / \mathrm{dl})$ & $7 \pm 2.8$ & $8.1 \pm 2.7$ & NS & $7.6 \pm 3.2$ & $7.4 \pm 2.2$ & NS & $7.5 \pm 3.1$ & $7.6 \pm 2.3$ & NS \\
\hline $\mathrm{EF}(\%)$ & $41.8 \pm 11.4$ & $41.7 \pm 12$ & NS & $41.67 \pm 11.3$ & $41.9 \pm 12.2$ & NS & $41.4 \pm 11.3$ & $42.4 \pm 12.2$ & NS \\
\hline NYHA class 2 & $38(67.9 \%)$ & $34(72.3 \%)$ & NS & $40(64.5 \%)$ & $32(78.0 \%)$ & NS & $43(65.2 \%)$ & $29(78.4 \%)$ & NS \\
\hline NYHA class 3 & $18(32.1 \%)$ & $13(27.7 \%)$ & & $22(35.5 \%)$ & $9(22.0 \%)$ & & $23(34.8 \%)$ & $8(21.6 \%)$ & \\
\hline
\end{tabular}

$C I$ collapse index, $I V C$ inferior vena cava, $C C$ caval congestion, $E F$ ejection fraction, $N S$ not significance

ROC curve analysis for predicting caval congestion identified the following cutoff: BUN/Cr of 25.5 (ROC 0.64; $95 \%$ CI $0.54-0.73$, sensitivity $59 \%$, specificity $70.3 \%$ ), BUN of 39.2 (ROC $0.61 ; 95 \%$ CI $0.51-0.70$, sensitivity $56.4 \%$, specificity $65.6 \%$ ) and eGFR of 45.8 (ROC 0.63 ; $95 \%$ CI $0.53-0.72$, sensitivity $87.2 \%$, specificity $46.9 \%$ ). ROC curve analysis for predicting impaired CI identified BUN/Cr of 23.7 (ROC 0.68; $95 \%$ CI 0.58-0.76, sensitivity $68.1 \%$, specificity $71.4 \%$ ), BUN of 31.8 (ROC 0.60; $95 \%$ CI $0.50-0.69$, sensitivity $78.7 \%$, specificity $41.1 \%$ ) and eGFR of 45.8 (ROC 0.55 ; $95 \%$ CI 0.45-0.65, sensitivity $78.7 \%$, specificity $44.6 \%$ ). Finally, ROC analysis for predicting impaired dilated IVC identified BUN/Cr of 25 (ROC $0.61 ; 95 \%$ CI $0.51-0.71$, sensitivity $58.5 \%$, specificity $66.1 \%$ ), BUN of 33.2 (ROC $0.60 ; 95 \%$ CI $0.50-0.70$, sensitivity $78 \%$, specificity $45.2 \%$ ) and eGFR of 45.8 (ROC 0.61 ; $95 \%$ CI $0.51-0.71$, sensitivity $80.5 \%$, specificity $43.5 \%)$.

We performed univariate analysis and multiple logistic regression analysis for risk of caval congestion, impaired $\mathrm{CI}$ and dilated IVC including in the model only variables found to be significant (Table 2). BUN/Cr $>25.5$ (adjusted OR 2.98, $p$ 0.015) and eGFR $\leq 45.8$ (adjusted OR 5.38, $p$ 0.002) were independently associated with risk of caval congestion; BUN/Cr $>23.7$ was the best predictor of impaired CI (adjusted OR 4.41, $p$ 0.001) and eGFR $\leq 45.8$ was the best predictive factor of dilated IVC (adjusted OR 2.73, $p$ 0.037) (Table 3).

\section{BUN/Cr and outcome}

Overall, 51 patients $(49.5 \%)$ died during the follow-up (median 31 months). The Kaplan-Meier curve for allcause death confirmed only BUN/Cr $>25.5$, other than NYHA class (data not shown), as a parameter significantly associated with reduced survival (Fig. 1).
By Cox time-dependent multivariate analysis including left ventricular EF, NYHA functional class, serum sodium, systolic blood pressure, metrics of renal function (BUN, creatinine, eGFR, uric acid, BUN/Cr) and caval congestion, we find that a BUN/Cr $>25.5$ ( HR 2.19, $95 \% \mathrm{CI}$ $1.21-3.94, p<0.001)$ and NYHA class 3 (HR 2.91, $95 \%$ CI $1.60-5.31, p<0.0005)$ are the only independent risk factors predicting risk of mortality.

\section{Discussion}

RD develops in approximately $50 \%$ of HF patients with normal renal function at the initial work-up, and it is a strong predictor of morbidity and mortality [17, 18]. Traditionally, renal impairment in HF is assumed to be mainly due to a decrease in cardiac output [2]. However, it is not rare to see a progression of $\mathrm{RD}$ in $\mathrm{HF}$ patients with preserved systolic function or with stable EF over time. Indeed, recent studies demonstrate that venous congestion may be the most important hemodynamic cause leading to RD in acute and chronic HF [19-21]. Most of these studies were conducted in patients who underwent cardiac catheterization for decompensated HF. Thus, the relation between venous congestion and renal function in outpatients setting remains to be verified. In this retrospective study, we sought to investigate in outpatients with HF and $\mathrm{RD}$ the relationship between routine renal metrics and noninvasive markers for the intravascular volume status evaluated by ultrasonography of IVC. The results show that such metrics of renal function (BUN/Cr, BUN and eGFR) are significantly deteriorated in patients with positive indices for caval congestion than patients without, suggesting that the worsening of the renal function may affect congestion and vice versa. Indeed, other studies underline that both heart and kidney worsen, in turn, the function of 
Table 3 Relationship between metrics of renal function and parameters of inferior vena cava

\begin{tabular}{|c|c|c|c|c|c|}
\hline & No CC $(n=64)$ & $\mathrm{CC}(n=39)$ & Crude OR (95\% CI) & Adjusted OR (95 \% CI) & $p$ \\
\hline \multicolumn{6}{|l|}{$\mathrm{BUN} / \mathrm{Cr}$} \\
\hline$\leq 25.5$ & $45(70.3 \%)$ & $16(41 \%)$ & 1 & 1 & \\
\hline$>25.5$ & $19(29.7 \%)$ & $23(59 \%)$ & $3.41(1.48-7.84)$ & $2.98(1.23-7.18)$ & 0.015 \\
\hline \multicolumn{6}{|c|}{ BUN (mg/dl) } \\
\hline$\leq 39.2$ & $42(65.6 \%)$ & $17(43.6 \%)$ & 1 & 1 & \\
\hline$>39.2$ & $22(34.4 \%)$ & $22(56.4 \%)$ & $2.47(1.09-5.59)$ & $0.74(0.26-2.14)$ & 0.6 \\
\hline \multicolumn{6}{|c|}{ eGFR_MDRD } \\
\hline$>45.8$ & $30(46.9 \%)$ & $5(12.8 \%)$ & 1 & 1 & \\
\hline \multirow[t]{2}{*}{$\leq 45.8$} & $34(53.1 \%)$ & $34(87.2 \%)$ & $6.00(2.08-17.31)$ & $5.38(1.82-15.89)$ & 0.002 \\
\hline & $\begin{array}{l}\mathrm{CI} \geq 50 \% \\
(n=56)\end{array}$ & $\begin{array}{l}\mathrm{CI}<50 \% \\
(n=47)\end{array}$ & $\begin{array}{l}\text { Crude OR (95\% } \\
\text { CI) }\end{array}$ & $\begin{array}{l}\text { Adjusted OR (95\% } \\
\text { CI) }\end{array}$ & $p$ \\
\hline \multicolumn{6}{|l|}{$\mathrm{BUN} / \mathrm{Cr}$} \\
\hline$\leq 23.7$ & $41(73.2 \%)$ & $17(36.2 \%)$ & 1 & 1 & \\
\hline$>23.7$ & $15(26.8 \%)$ & $30(63.8 \%)$ & $4.82(2.08-11.16)$ & $4.41(1.87-10.37)$ & 0.001 \\
\hline \multicolumn{6}{|c|}{ BUN (mg/dl) } \\
\hline$\leq 31.8$ & $25(44.6 \%)$ & $12(25.5 \%)$ & 1 & 1 & \\
\hline$>31.8$ & $31(55.45)$ & $35(74.5 \%)$ & $2.35(1.01-5.45)$ & $0.38(0.11-1.36)$ & 0.14 \\
\hline \multicolumn{6}{|c|}{ eGFR_MDRD } \\
\hline$>45.8$ & $25(44.6 \%)$ & $10(21.3 \%)$ & 1 & 1 & \\
\hline \multirow[t]{2}{*}{$\leq 45.8$} & $31(55.4 \%)$ & $37(78.7 \%)$ & $2.98(1.24-7.16)$ & $2.55(1.01-6.44)$ & 0.049 \\
\hline & $\begin{array}{l}\text { IVCED } \leq 2 \mathrm{~cm} \\
(n=62)\end{array}$ & $\begin{array}{l}\text { IVCED }>2 \mathrm{~cm} \\
(n=41)\end{array}$ & $\begin{array}{l}\text { Crude OR (95\% } \\
\text { CI) }\end{array}$ & $\begin{array}{l}\text { Adjusted OR (95\% } \\
\text { CI) }\end{array}$ & $p$ \\
\hline \multicolumn{6}{|l|}{$\mathrm{BUN} / \mathrm{Cr}$} \\
\hline$\leq 25$ & $39(62.9 \%)$ & $16(39.0 \%)$ & 1 & 1 & \\
\hline$>25$ & $23(37.1 \%)$ & $25(61.0 \%)$ & $2.65(1.18-5.97$ & $2.26(0.98-5.22)$ & 0.056 \\
\hline \multicolumn{6}{|c|}{ BUN (mg/dl) } \\
\hline$\leq 33.2$ & $25(40.3 \%)$ & $8(19.5 \%)$ & 1 & 1 & \\
\hline$>33.2$ & $37(59.7 \%)$ & $33(80.5 \%)$ & $2.79(1.11-7.02$ & $1.03(0.29-3.65)$ & 0.9 \\
\hline \multicolumn{6}{|c|}{ eGFR_MDRD } \\
\hline$>45.8$ & $27(43.5 \%)$ & $8(19.5 \%)$ & 1 & 1 & \\
\hline$\leq 45.8$ & $35(56.5 \%)$ & $33(80.5 \%)$ & $3.18(1.27-7.99$ & $2.73(1.06-7.02)$ & 0.037 \\
\hline
\end{tabular}

$C C$ caval congestion, $C I$ collapse index, IVCED maximum expiratory diameter of inferior vena cava, $O R$ odds ratio the other, thereby perpetuating the vicious cycle of congestive HF [22].

Both renal function and congestion represent the intersection between the patient's specific clinical HF profile, treatment, severity of disease, intrinsic kidney illness and co-morbid conditions. The net amalgamation of these characteristics is what produces the observed renal function and congestion state across populations. Thus, any associations we find may be cause and effect from the congestion or kidney disease [23]. In our study, multiple logistic regression analysis indicates that BUN/Cr $>25.5$ and eGFR $\leq 45.8$ are significantly associated with an elevated risk for caval congestion (dilatation and impaired collapsibility of IVC) despite their areas under ROC curve being less than 0.70 . This fact can be explained because
BUN/Cr and eGFR, although having reduced diagnostic accuracy, are significant predicting factors for caval congestion in a multivariate model. Thus, considering that the primary mediator of increased venous tone is neurohormonal activation, any association between renal function and caval congestion may reflect not merely hypervolemia, but also the neurohormonal status of the patient and the response to neurohormonal blocking therapies. On the basis of this consideration, we can explain why $\mathrm{BUN} / \mathrm{Cr}$ is a stronger biomarker of caval congestion than eGFR. Moreover, in our study, serum creatinine, as well as uric acid, is not related with IVC parameters, suggesting a poor utility in predicting congestion-related RD. These results may aid to clarify recent data that reduce the prognostic role of creatinine in $\mathrm{HF}$ 
Fig. 1 Kaplan-Meier analysis for all-cause death according to $\mathrm{BUN} / \mathrm{Cr}$

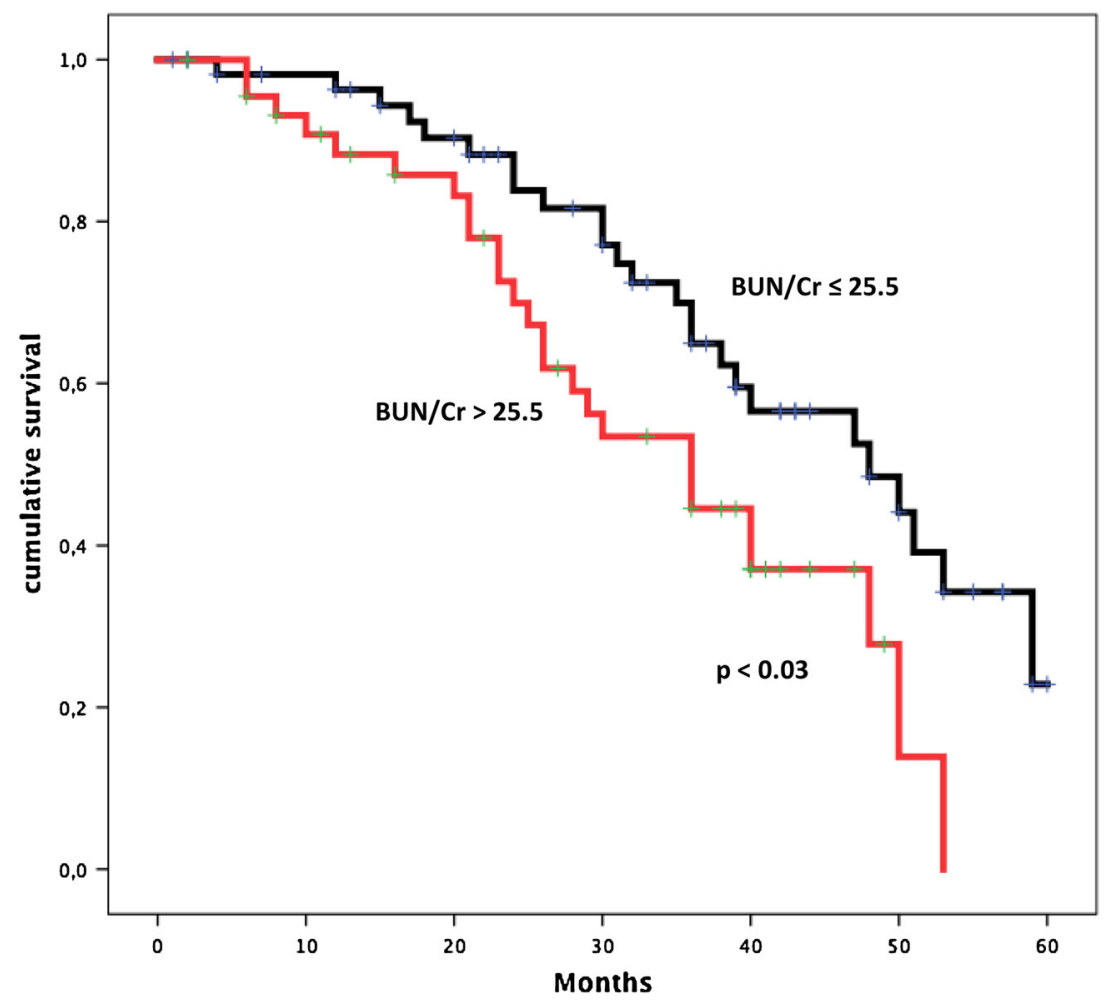

[24] supporting the role of $\mathrm{BUN}$ and $\mathrm{BUN} / \mathrm{Cr}$ as better indicators of outcome [25].

Indeed, our study confirms BUN/Cr as a powerful predictor of long-term all-cause mortality in outpatients with stable $\mathrm{HF}$, while Brisco et al. find BUN/Cr associated with a worsened survival in the setting of decompensated $\mathrm{HF}$ [14]. This result may be explained by the fact that BUN/Cr incorporates the cumulative effects of several influences, including hemodynamic alterations resulting in renal hypoperfusion, and the extent of neurohormonal activation, which is closely associated with altered renal hemodynamics [26] and the progression of cardiac failure [27]. HF causes arterial underfilling resulting in activation of the sympathetic nervous system (SNS), renin-angiotensin-aldosterone system (RAAS) and non-osmotic arginine vasopressin (AVP) release [13, 28]. SNS and RAAS activation cause increased reabsorption of sodium and water in the proximal tubule, leading to increased proximal tubular urea concentration in the collecting duct, with resulting increased urea reabsorption [29]. Elevated AVP levels increase urea reabsorption in the collecting duct without creatinine reabsorption [30]. In the absence of conditions that increase urea production, elevations in BUN level are often determined by a decrease in glomerular filtration rate [21]. Moreover, urea reabsorption in proximal tubules (40-50\% of filtered urea) is passive and is linked to the reabsorption of sodium and water [31]. Thus, the increase in sodium reabsorption in HF produces a parallel increase in urea reabsorption. The net result is a reduced urea excretion and an elevation in BUN level that is not solely due to the fall in glomerular filtration rate, and, therefore, not associated with a proportional rise in serum creatinine level [32].

The results demonstrate that patients with higher BUN/ $\mathrm{Cr}$ or lower eGFR have greater risk of caval congestion, and, for this reason, they should receive close follow-up. In addition, patients with a higher BUN/Cr also have an increased risk of mortality in the long term. Physicians should ensure these high-risk patients receive clinical surveillance and an optimized, evidence-based HF regimen to reduce their risk of subsequent morbidity and mortality.

The prognostic meaning of uric acid in HF and its relationship with renal worsening are well known in the literature and are similar to that of BUN. In this study, uric acid is not associated with IVC ultrasonographic metrics or mortality. This result might have been influenced by the fact that $50 \%$ of study population were in treatment with allopurinol.

\section{Conclusion}

In conclusion, the results of this study provide evidence that, in outpatients with $\mathrm{HF}$ and concomitant RD, BUN/Cr and eGFR are routine renal metrics significantly associated with caval congestion, defined by ultrasonography. These 
data, thereby confirming in an ambulatory setting previous findings regarding congestion and kidney function in $\mathrm{HF}$, suggest these parameters as surrogate biomarkers of cardiorenal decompensation and HF-related hemodynamic and neurohormonal alterations. Moreover, BUN/Cr, the only renal marker associated with all-cause mortality with NYHA class, may be considered an easily accessible tool for stratification of HF severity in these patients. Considering the intrinsic limitations related to a single-centre retrospective study and a small sample size, additional investigations are required to confirm these findings, and in order to further understand the intricate bidirectional relation occurring between renal function and venous congestion.

Conflict of interest The authors report no relationships that could be construed as a conflict of interest.

Statement of human and animal rights All procedures performed in human participants were in accordance with the ethical standards of the institutional research committee and with the 1964 Helsinki declaration and its later amendments or comparable ethical standards. This article does not contain any studies with animals performed by any of the authors.

Informed consent Informed consent was obtained from all individual participants included in the study.

\section{References}

1. Cioffi G, Mortara A, Di Lenarda A, Oliva F, Lucci D, Senni M, Cacciatore G, Chinaglia A, Tarantini L, Metra M, Maggioni AP, Tavazzi L, on behalf of Italian Acute Heart Failure Survey (2013) Clinical features, and in-hospital and 1-year mortalities of patients with acute heart failure and severe renaldysfunction. Data from the Italian Registry IN-HF Outcome. Int J Cardiol pii: S0167-5273(13)01074-7

2. Ljungman S, Laragh JH, Cody RJ et al (1990) Role of the kidney in congestive heart failure: relationship of cardiac index to kidney function. Drug 39:10-24

3. Mullens W, Abrahams Z, Francis GS et al (2009) Importance of venous congestion for worsening of renal function in advanced decompensated heart failure. J Am Coll Cardiol 53:589-596

4. Sinkeler SJ, Damman K, van Veldhuisen DJ, Hillege H, Navis G (2012) A re-appraisal of volume status and renal function impairment in chronic heart failure: combined effects of pre-renal failure and venous congestion on renal function. Heart Fail Rev 17:263-270

5. Parrinello G, Torres D, Paterna S, Di Pasquale P, Licata G (2008) The pathophysiology of acute heart failure: the key role of fluid accumulation. Am Heart J 156:e19

6. Dupont M, Mullens W, Tang WH (2011) Impact of systemic venous congestion in heart failure. Curr Heart Fail Rep 8:233-241

7. Temporelli PL, Scapellato F, Giannuzzi P (2000) Non-invasive evaluation of the hemodynamic profile in patients with heart failure: estimation of right atrial pressure. Ital Heart J 1:1317S$1320 \mathrm{~S}$

8. Hollerbach S, Schultze K, Muscholl M, Schölmerich J (2001) Ultrasonography of the inferior vena cava (IVC) in the diagnosis and monitoring of therapy in patients with chronic congestive heart failure. Dtsch Med Wochenschr 126:129-133

9. Feissel M, Michard F, Faller JP et al (2004) The respiratory variation in inferior vena cava diameter as a guide to fluid therapy. Intensive Care Med 30:1834-1837

10. Blehar DJ, Dickman E, Gaspari R (2009) Identification of congestive heart failure via respiratory variation of inferior vena cava diameter. Am J Emerg Med 27:71-75

11. McMurray JJ, Adamopoulos S, Anker SD, Auricchio A, Böhm M, Dickstein K, Falk V, Filippatos G, Fonseca C, Gomez-Sanchez MA, Jaarsma T, Køber L, Lip GY, Maggioni AP, Parkhomenko A, Pieske BM, Popescu BA, Rønnevik PK, Rutten FH, Schwitter J, Seferovic P, Stepinska J, Trindade PT, Voors AA, Zannad F, Zeiher A, ESC Committee for Practice Guidelines (2012) ESC Guidelines for the diagnosis and treatment of acute and chronic heart failure 2012: the task force for the diagnosis and treatment of acute and chronic heart failure 2012 of the European Society of Cardiology. Developed in collaboration with the Heart Failure Association (HFA) of the ESC. Eur Heart J 33(14): 1787-1847

12. Levey AS, Coresh J, Greene T, Stevens LA, Zhang YL, Hendriksen S, Kusek JW, Van Lente F, Chronic Kidney Disease Epidemiology Collaboration (2006) Using standardized serum creatinine values in the modification of diet in renal disease study equation for estimating glomerular filtration rate. Ann Intern Med 145:247-254

13. Lin HJ, Chao CL, Chien KL, Ho YL, Lee CM, Lin YH, Wu YW, Hsu RB, Chou NK, Wang SS, Chen CY, Chen MF (2009) Elevated blood urea nitrogen-to-creatinine ratio increased the risk of hospitalization and all-cause death in patients with chronic heart failure. Clin Res Cardiol 98:487-492

14. Brisco MA, Coca SG, Chen J, Owens AT, McCauley BD, Kimmel SE, Testani JM (2013) Blood urea nitrogen/creatinine ratio identifies a high-risk but potentially reversible form of renal dysfunction in patients with decompensated heart failure. Circ Heart Fail 6:233-239

15. Beigel R, Cercek B, Luo H, Siegel RJ (2013) Noninvasive evaluation of right atrial pressure. J Am Soc Echocardiogr. doi:10.1016/j.echo.2013.06.004

16. Kircher BJ, Himelman RB, Schiller NB (1990) Noninvasive estimation of right atrial pressure from the inspiratory collapse of the inferior vena cava. Am J Cardiol 66:493-496

17. Santoro A (2012) Heart failure and cardiorenal syndrome in the elderly. J Nephrol 25(Suppl 19):S67-S72

18. McCullough PA, Kellum JA, Haase M, Müller C, Damman K, Murray PT, Cruz D, House AA, Schmidt-Ott KM, Vescovo G, Bagshaw SM, Hoste EA, Briguori C, Braam B, Chawla LS, Costanzo MR, Tumlin JA, Herzog CA, Mehta RL, Rabb H, Shaw AD, Singbartl K, Ronco C (2013) Pathophysiology of the cardiorenal syndromes: executive summary from the eleventh consensus conference of the Acute dialysis quality initiative (ADQI). Contrib Nephrol 182:82-98

19. Damman K, Navis G, Smilde TD et al (2007) Decreased cardiac output, venous congestion and the association with renal impairment in patients with cardiac dysfunction. Eur J Heart Fail 9:872-878

20. Damman K, Voors AA, Hillege HL et al (2010) Congestion in chronic systolic heart failure is related to renal dysfunction and increased mortality. Eur J Heart Fail 12:974-982

21. Damman K, van Deursen VM, Navis G et al (2009) Increased central venous pressure is associated with impaired renal function and mortality in a broad spectrum of patients with cardiovascular disease. J Am Coll Cardiol 53:582-588

22. Parrinello G, Greene SJ, Torres D, Alderman M, Bonventre JV, Di Pasquale P, Gargani L, Nohria A, Fonarow GC, Vaduganathan M, Butler J, Paterna S, Stevenson LW, Gheorghiade M (2014) 
Water and sodium in heart failure: a spotlight on congestion. Heart Fail Rev 20:13-24

23. Testani JM, Damman K (2013) Venous congestion and renal function in heart failure. it's complicated. Eur J Heart Fail 15:599-601

24. Kazory A (2010) Emergence of blood urea nitrogen as a biomarker of neurohormonal activation in heart failure. Am J Cardiol 106:694-700

25. Aronson D, Mittleman MA, Burger AJ (2004) Elevated blood urea nitrogen level as a predictor of mortality in patients admitted for decompensated heart failure. Am J Med 116:466-473

26. Martin PY, Schrier RW (1997) Sodium and water retention in heart failure: pathogenesis and treatment. Kidney Int Suppl 59(Suppl):S57-S61

27. Packer M (1992) The neurohormonal hypothesis: a theory to explain the mechanism of disease progression in heart failure. J Am Coll Cardiol 20:248-254
28. Parrinello G, Torres D, Paterna S (2011) Salt and water imbalance in chronic heart failure. Intern Emerg Med Suppl 1:29-36

29. Testani JM, Coca SG, Shannon RP, Kimmel SE, Cappola TP (2011) Influence of renal dysfunction phenotype on mortality in the setting of cardiac dysfunction: analysis of three randomized controlled trials. Eur J Heart Fail 13:1224-1230

30. Bankir LT, Trinh-Trang-Tan MM (2000) Renal urea transporters. Direct and indirect regulation by vasopressin. Exp Physiol $85 \mathrm{~S}: 243 \mathrm{~s}-252 \mathrm{~s}$

31. Francis GS, Siegel RM, Goldsmith SR et al (1985) Acute vasoconstrictor response to intravenous furosemide in patients with chronic congestive heart failure. Activation of the neurohumoral axis. Ann Intern Med 103:1-6

32. Dossetor JB (1966) Creatininemia versus uremia. The relative significance of blood urea nitrogen and serum creatinine concentrations in azotemia. Ann Intern Med 65:1287-1299 